\title{
Characterization of Two Forms of Acetolactate Synthase from Barley
}

\author{
Jong-Mo Yoon, Moon-Young Yoon ${ }^{\dagger}$, Young Tae Kim* and Jung-Do Choi* \\ School of Life Sciences and Biotechnology Research Institute, Chungbuk National University, Cheongju 361-763, Korea \\ 'Department of Chemistry, Hanyang University, Seoul 133-791, Korea \\ Department of Microbiology, Pukyong National University, Pusan 608-737, Korea
}

Received 24 February 2003, Accepted 13 March 2003

\begin{abstract}
Acetolactate synthase (ALS) catalyzes the first common step in the biosynthesis of valine, leucine, and isoleucine. ALS is the target site for several classes of herbicides, including sulfonylureas, imidazolinones, and triazolopyrimidines. Two forms of ALS (designated ALS I and ALS II) were separated from barley shoots by heparin affinity column chromatography. The molecular masses of native ALS I and ALS II were determined to be $248 \mathrm{kDa}$ and $238 \mathrm{kDa}$ by nondenaturing gel electrophoresis and activity staining. Similar molecular masses of two forms of ALS were confirmed by a Western blot analysis. SDSPAGE and Western blot analysis showed that the molecular masses of the ALS I and ALS II subunits were identical - $65 \mathrm{kDa}$. The two ALS forms exhibited different properties with respect to the values of $K_{m}, p I$ and optimum pH, and sensitivity to inhibition by herbicides sulfonylurea and imidazolinone as well as to the feedback regulation by the end-product amino acids Val, Leu, and Ile. These results, therefore, suggest that the two ALS forms are not different polymeric forms of the same enzyme, but isozymes.
\end{abstract}

Keywords: Acetolactate synthase, Affinity, Barley, Herbicides, Heparin, Isozyme

\section{Introduction}

Acetolactate synthase (ALS, EC 4.1.3.18 : also referred to as acetohydroxyacid synthase) catalyzes the first common step of a bifurcated biosynthetic pathway to the branched-chain amino acids, valine, leucine, and isoleucine in bacteria, yeast, and higher plants. ALS catalyzes either the condensation of 2acetolactate from two molecules of pyruvate in the first step of

\footnotetext{
*To whom correspondence should be addressed.

Tel: 82-43-261-2308; Fax: 82-43-267-2306

E-mail: jdchoi@cbucc.chungbuk.ac.kr
}

the valine and leucine synthetic pathway, or the formation of 2-aceto-2-hydroxybutyrate from pyruvate and 2-ketobutyrate as the second step of isoleucine biosynthesis. ALS is a key controlling point for the levels of the branched-chain amino acids in both prokaryotes and eukaryotes. In bacteria, this control involves the feedback inhibition of the enzyme activity by the branched-chain amino acids or repression of enzyme synthesis or both (Chipman et al., 1988). In plants, however, the only known mechanism of the regulation of ALS is through a feedback inhibition by valine, leucine, and isoleucine (Miflin, 1971).

ALS has received special attention in recent years since it was discovered that several classes of herbicides, including sulfonylureas (LaRossa and Schloss, 1984; Ray, 1984), imidazolinones (Shaner et al., 1984), and triazolopyrimidines (Kleschick et al., 1990; Namgoong et al., 1999), exhibit herbicidal activity by inhibiting this enzyme. ALS-inhibiting herbicides do not act as analogs of the substrates and cofactors, suggesting that the inhibition mechanism is complex. The most active ALS research areas are the structural studies of the herbicide binding site, as well as herbicide resistant mutations. Recently, site-directed mutagenesis studies in our laboratory revealed that in tobacco ALS, Trp-563 (Chong et al., 1999), Ala-121 and Ser-652 (Chong and Choi, 2000), and Lys-255 (Yoon et al., 2002) residues are probably involved in the binding of herbicides.

In bacteria, three ALS isozymes that differ in substrate preference and feedback regulation have been purified and characterized well (Grimminger and Umbarger, 1979: Schloss et al., 1985). Each of the isozymes is composed of two catalytic subunits of approximately $60 \mathrm{kDa}$ as well as two smaller, regulatory subunits ( 9 to $17 \mathrm{kDa}$ ) (Grimminger and Umbarger, 1979; Eoyang and Silverman, 1984; Schloss et al., 1985; Joo and Kim, 2001). In plants, the quaternary structure of ALS appears more diverse since the molecular mass of the nondenatured ALS enzymes varies significantly from $55 \mathrm{kDa}$ to $440 \mathrm{kDa}$ in different species (Duggleby and Pang, 2000). There has been speculation on the presence of different isozymes in plants, based on the differential sensitivity of the 
enzyme to inhibition by the branched-chain amino acids at different $\mathrm{pH}$ values (Miflin, 1971; Davis et al., 1977). Singh et al. (1988) first reported that ALS from Black Mexican Sweet corn cell cultures yielded two active peaks with different enzymatic properties by MonoQ chromatography. Recently, Shin et al. (1999) reported the separation of two ALS forms from peas that had different native molecular masses and exhibited different properties with respect to kinetic parameters and sensitivity to inhibition by the branched-chain amino acids. In this report, we provide evidence that the two ALS forms from barley are not different oligomeric forms of the same enzyme, but isozymes.

\section{Materials and Methods}

Materials Sodium pyruvate, thiamine pyrophosphate (TPP), flavin adenine dinucleotide (FAD), dithiothreitol (DTT), L-leucine, L-valine, L-isoleucine, $\alpha$-naphthol, creatine, ethylenediamine tetraacetic acid (EDTA), dimethyl sufoxide (DMSO), and Sephadex G-25 were purchased from the Sigma Chemical Co. (St. Louis, USA). DEAE-Sephacel was obtained from Pharmacia Biotech (Uppsala, Sweden). Heparin was the product of Bio-Rad Laboratories (Hercules, USA). A series of isoelectric focusing agents were from NOVEX (St. Roselle, USA). Herbicides, Londax, and Cadre were provided by Dr. Dae-Whang Kim (Korea Research Institute of Chemical Technology, Daejeon, Korea). The anti-ALS antibody against recombinant tobacco ALS-GST was provided by Dr. Chom Kyu Chong (Asan Biotech Institute, Kyunggi Do, Korea). The ALS-antibody-antibody-labeled HRP (horseradish peroxidase) was obtained from Amersham Co. (Arlington, USA).

Partial purification of acetolactate synthase from barley Barley seeds were sown in vermiculate. The shoots were grown in a dark room at $25^{\circ} \mathrm{C}$ for $7 \mathrm{~d}$ and then harvested. All of the other procedures were performed at $4^{\circ} \mathrm{C}$ with minimum exposure to light, usually $150 \mathrm{~g}$ barley shorts were homogenized in a volume of $600 \mathrm{ml}$ of a standard buffer $(50 \mathrm{mM}$ Tris- $\mathrm{HCl} \mathrm{pH} 7.8,15 \%$ glycerol, $1 \mathrm{mM}$ EDTA, $1 \mathrm{mM}$ DTT) that contained $50 \mathrm{mM} \mathrm{FAD,} 5 \mathrm{mM} \mathrm{MgCl}_{2}$, and $5 \mathrm{mM}$ L-leucine. The shoot was homogenized in a blender for $280 \mathrm{~s}$ $(7 \times 40 \mathrm{~s})$. The homogenate was filtered through eight layers of cheese cloth and centrifuged for $10 \mathrm{~min}$ at 20,000 $\mathrm{g}$. Solid ammonium sulfate was added to the supernatant (30\% saturation, $1.76 \mathrm{~g} / \mathrm{ml}$ ) and the precipitate was removed by centrifugation for $30 \mathrm{~min}$ at $24,000 \mathrm{~g}$. The supernatant was treated to a $45 \%$ saturation of ammonium sulfate and centrifuged for $30 \mathrm{~min}$ at $24,000 \mathrm{~g}$. The precipitate was resuspended in a minimum volume of a standard buffer and desalted on a Sephadex G-25 column. The desalted sample was loaded onto the DEAE-Sephacel column that was equilibrated with a standard buffer. The column was washed with the same buffer until the absorbance of eluent at $280 \mathrm{~nm}$ was near 0 , and the bound proteins were eluted with a linear gradient of 0 to $350 \mathrm{mM} \mathrm{NaCl}$. The active fractions were pooled and then concentrated by ultrafiltration using Amicon-cell with PM-30 membrane. The protein solution was loaded onto a heparin-affinity column $(2.5 \times 10 \mathrm{~cm})$ that was equilibrated with a $25 \mathrm{mM}$ Tris- $\mathrm{HCl}$ $(\mathrm{pH} 7.45)$ buffer that contained $15 \%$ glycerol, $1 \mathrm{mM} \mathrm{MgCl}_{2}$,
$0.5 \mathrm{mM}$ DTT, and $0.05 \mathrm{mM}$ EDTA. A part of the ALS activity was found in the unbound fractions of the sample. Elution of the heparin-bound proteins was carried out with a Tris buffer that contained $500 \mathrm{mM} \mathrm{NaCl}$. The fractions that contained ALS activity were stored in liquid nitrogen.

Enzyme assay The enzyme activity was measured according to the method of Westerfeld (1945), as modified by Ray (1984). The assay mixture contained $20 \mathrm{mM}$ potassium phosphate buffer $(\mathrm{pH}$ 7.0), $0.5 \mathrm{mM}$ TPP, $10 \mu \mathrm{m}$ FAD, $20 \mathrm{mM}$ pyruvate, $20 \mathrm{mM} \mathrm{MgCl}_{2}$, plus enzyme in a final volume of $200 \mu$ l. After incubation at $37^{\circ} \mathrm{C}$ for $60 \mathrm{~min}$, the enzyme reaction was stopped by the addition of $20 \mu \mathrm{l}$ of $6 \mathrm{~N} \mathrm{H}_{2} \mathrm{SO}_{4}$. Acetolactate, the enzyme reaction product, was allowed to decarboxylate at $60^{\circ} \mathrm{C}$ for $15 \mathrm{~min}$. The acetoin that was formed by acidification was incubated with $200 \mu \mathrm{l}$ of $0.5 \%$ creatine and $200 \mu \mathrm{l}$ of $5 \% \alpha$-naphthol at $60^{\circ} \mathrm{C}$ for $15 \mathrm{~min}$. The absorbance of the reaction mixture was measured at $525 \mathrm{~nm}$. The protein concentration was determined by the method of Bradford (1976).

Gel electrophoresis SDS-PAGE was performed on $11 \%$ acrylamide gel that contained $0.1 \%$ SDS. Nondenaturing gel electrophoresis was carried out according to the method of Laemmli (1970) without SDS in 5\%, 6\%, 7\%, and 8\% acrylamide separating gels and a $4 \%$ stacking gel. Native isoelectric focusing was carried out on a $5 \%$ acrylamide gel in which the $\mathrm{pH}$ gradient was 3 to 10 . The gel contained $2 \%$ ampholytes without denaturing agents, and the focusing conditions were adapted from Robertson $e t$ al. (1987)

Activity staining Activity staining was performed as described by Grimminger and Umbarger (1970). After the nondenaturing gel electrophoresis, the gel was sliced into $2 \mathrm{~mm}$ pieces and incubated in the assay mixture for 1 to $3 \mathrm{~h}$ at $37^{\circ} \mathrm{C}$ and stained with creatine and $\alpha$-naphtol, as described in the enzyme assay.

Western blot analysis The samples were electrophoresed on the gel in the presence or absence of $0.1 \%$ SDS and transferred to a PVDF membrane using a TE-22 mini transfer electrophoresis unit. Hybridization was carried out with the first antibody (tobacco ALSGST antibody from rabbit) for a $1 \mathrm{~h}$-shaking incubation. The blots were washed out with the blocking solution. On this transferred membrane, the second antibody-labeled HRP (horseradish peroxidase) was hybridized. The membrane sheets were washed with $20 \mathrm{mM}$ Tris (pH 7.5) and $0.5 \mathrm{M} \mathrm{NaCl}$ that contained $0.05 \%$ Tween-20 and detected by the ECL method, described in the manufacturer's protocol.

\section{Results}

Separation of two of the ALS Acetolactate synthase was partially purified from the etiolated barley shoots by ammonium sulfate fractionation and DEAE-sephacel chromatography. Heparin-affinity chromatography resolved the partially-purified ALS into two peaks of activity (Fig. 1). The minor peak of activity (designated ALS I) did not bind to heparin. On the other hand, the major activity peak 


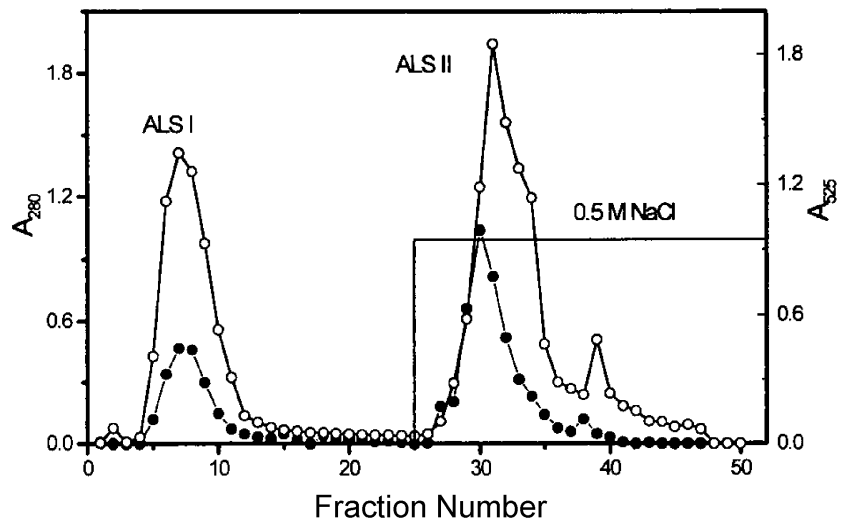

Fig. 1. Chromatography of acetolactate synthase on a heparin affinity column. Elution was carried out with the $0.5 \mathrm{M} \mathrm{NaCl}$. protein $(\bigcirc)$, enzyme activity
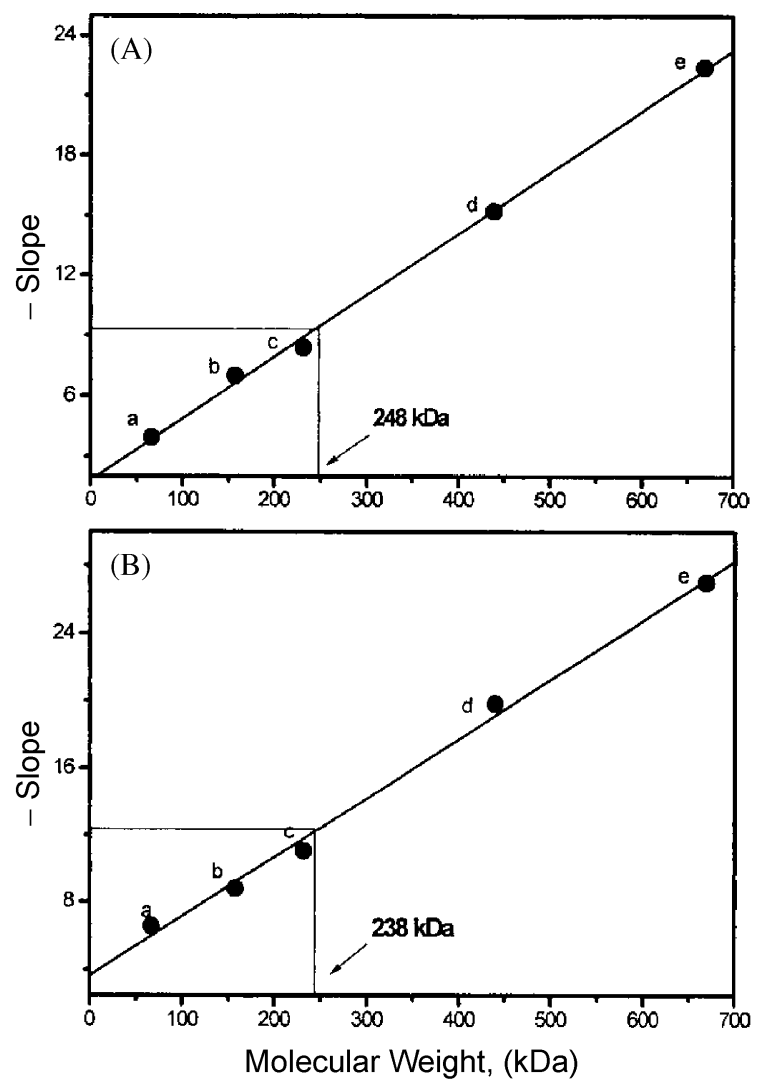

Fig. 2. Molecular mass determination of the active forms of ALSI (A) and ALSII (B) by nondenaturing gel electrophoresis and activity assay. Graph of -slope of $100 \times \log \left(\mathrm{R}_{\mathrm{f}} \times 100\right)$ versus gel concentration $(\%)$ as a function of the molecular mass. The slopes were obtained for the five proteins, which were a, BSA; b, aldolase; c, catalase; d, ferritin; and e, thyroglobulin.

(designated ALS II) was bound to heparin and eluted with 0.5 $\mathrm{M} \mathrm{NaCl}$. The two ALS forms were significantly different with respect to enzymatic properties and sensitivity to feedback inhibition by the end products, Val, Leu, Ile, and inhibition by herbicides, Londax and Cadre (see below for details).

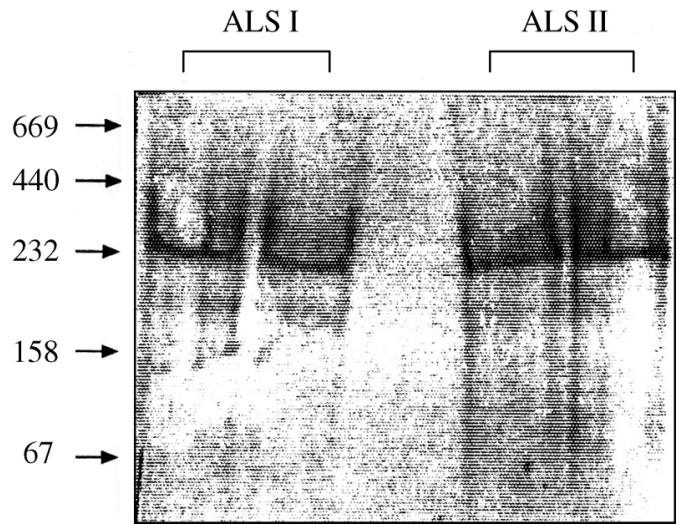

Fig. 3. Western blot analysis using anti-ALS antibody. After native PAGE, the proteins were transferred onto a PVDF membrane and detected by ECL.

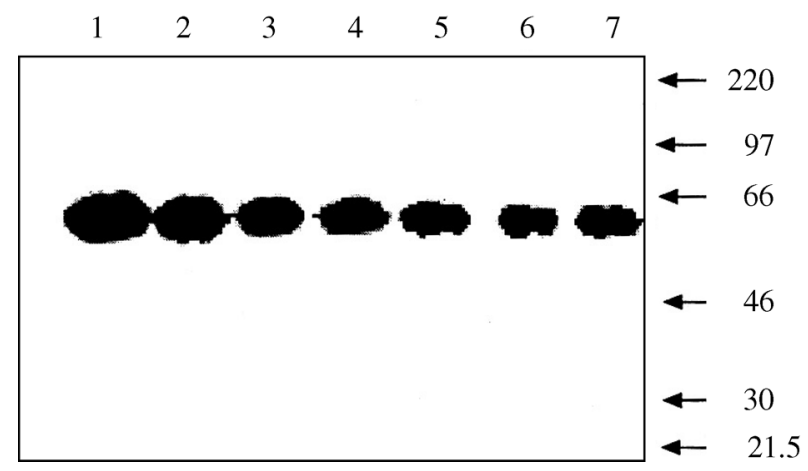

Fig. 4. Western blot analysis using anti-ALS antibody. After SDS-PAGE, the proteins were transferred onto a PVDF membrane and detected by ECL. Lane 1, homogenate extracts; lane 2, ammonium sulfate fractionation desalted with Sephadex G-25; lane 3, DEAE-Sephacel; lanes 4-5, ALSI; lanes 6-7, ALSII.

Molecular and subunit masses of ALS I and ALS II The molecular masses of the native forms of ALS I and ALS II were determined by nondenaturing gel electrophoresis and activity staining, according to the method of Grimminger and Umbarger (1979). The native molecular masses of ALS I and ALS II were estimated to be approximately $248 \mathrm{kDa}$ and 238 $\mathrm{kDa}$, respectively (Fig. 2). A Western blot analysis of the native PAGE of ALS I and ALS II using a polyclonal antiALS antibody, showed that the molecular masses of the native forms of both ALS I and ALS II are approximately 230-250 $\mathrm{kDa}$ (Fig. 3), which confirms the result that was obtained by the native PAGE and activity staining. The subunit mass for ALS I and ALS II was determined to be identical - $65 \mathrm{kDa}$ by SDS-PAGE, followed by a Western blot (Fig. 4). These results imply that the native forms of both ALS I and ALS II are likely tetrameric oligomers.

The enzymatic properties of ALS I and ALS II The physical and kinetic properties of ALS I and ALS II are 
Table 1. Summary of the physical and kinetic properties of ALS I and ALS II

\begin{tabular}{ccc}
\hline Forms of ALS & ALS I & ALS II \\
\hline Native molecular weight $(\mathrm{kDa})$ & 248 & 238 \\
Subunit molecular weight $(\mathrm{kDa})$ & 65 & 65 \\
$K_{m}$ for pyruvate $(\mathrm{mM})$ & 10 & 11 \\
pH optima & 7.5 & 7.0 \\
pI & 4.6 & 5.3 \\
\hline
\end{tabular}
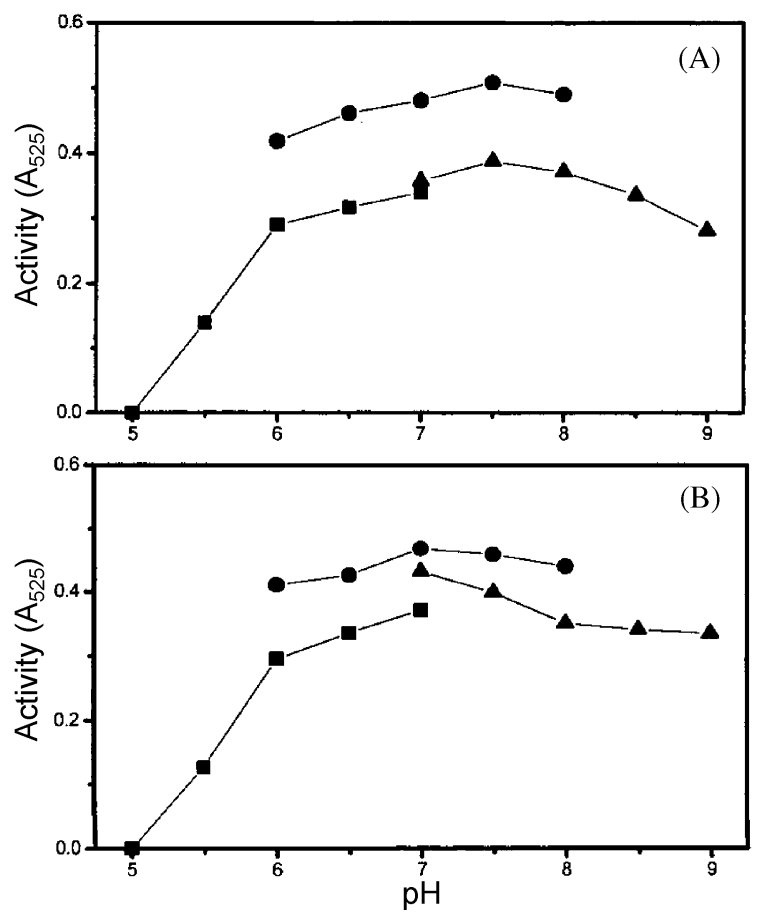

Fig. 5. The pH optimum of ALS I (A) and ALS II (B). The pH of the assay solution varied by using the indicated buffers. ( Citrate; O, Phosphate; $\boldsymbol{\Delta}$, Tris).

summarized in Table 1. The substrate saturation curves for both ALS I and ALS II showed a hyperbolic pattern, as reported previously (Shin et al. 1999, data not shown). Lineweaver-Burk plots of the data for ALS I and ALS II showed no significant deviation from the straight lines and gave the $K_{m}$ values of $10 \mathrm{mM}$ and $11 \mathrm{mM}$, respectively (data not shown). Both ALS I and ALS II were assayed over a $\mathrm{pH}$ range from 5.0 to 9.0 using three different buffer systems (citrate, phosphate, and Tris buffers). The activity-pH profiles for ALS I and ALS II showed a pH optima of 7.5 and 7.0 for ALS I and ALS II, respectively (Fig. 5). The pI values of ALS I and ALS II were determined to be 4.6 and 5.3, respectively, by isoelectric focusing and activity staining (Fig. 6). This indicates that ALS I is somewhat more acidic than ALS II. To determine the feedback inhibition of ALS by the end products, the two ALS forms were assayed in the presence of various concentrations of leucine, valine, isoleucine, and leucine plus

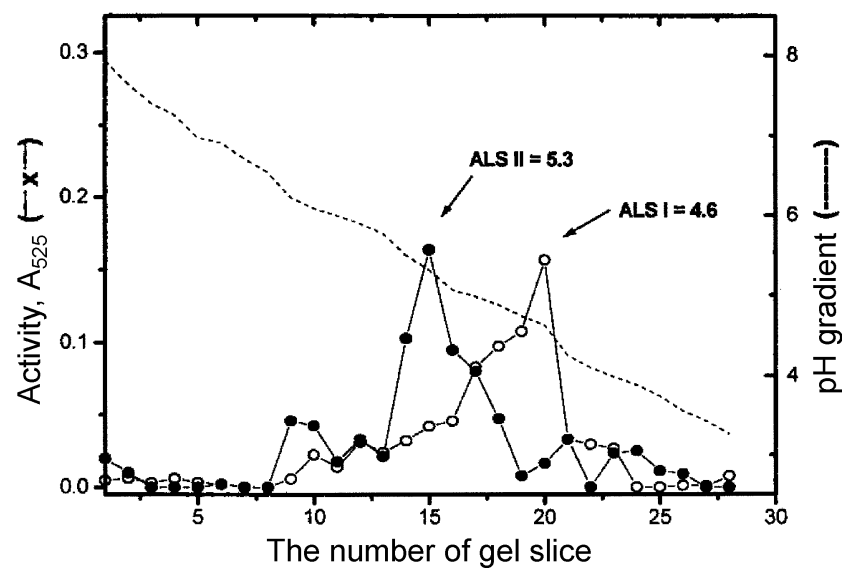

Fig. 6. Activity staining profiles of ALS I and ALS II after IEFgel electrophoresis. ALS I ( $\bigcirc)$, ALS II (○).

valine (Fig. 7). The results showed that ALS I was less inhibited than ALS II by these amino acids. Leucine was most effective in the inhibition of ALS II. The mixture of leucine and valine was more effective in the inhibition of both forms of ALS than any other single amino acid. The inhibition of ALS I and ALS II by imidazolinone Cadre and sulfonylurea Londax was also examined. ALS II was significantly more sensitive to both herbicides than ALS I (Fig. 8).

\section{Discussion}

Three different ALS isozymes, which were similar in structure but differed in enzymatic properties with respect to substrate specificity, kinetics, and feedback regulation, have been purified in bacteria (Grimminger and Umbarger, 1979; Schloss et al., 1985). Each of these isozymes is composed of two catalytic subunits of about $60 \mathrm{kDa}$ and two smaller, regulatory subunits (Grimminger and Umbarger, 1979; Eoyang and Silverman, 1984; Schloss et al., 1985). The primary sequences of the $\sim 60 \mathrm{kDa}$ subunits of the three isozymes show considerable homology with about a $40 \%$ amino acid identity between any pair. All of the ALS catalytic polypeptides from a variety of organisms were homologous, at least to these extents. The expression of the three ALS isozymes was regulated in different ways and had complementary roles (Barak et al., 1990).

The low abundance and labile nature of plant ALS have severely hampered the purification and characterization of the enzyme. Thus, the quaternary structure of plant ALS is far less clear than that of bacterial enzymes. Although the presence of the ALS isozymes in plants has long been speculated, this is still unclear. In this report, we present some biochemical evidence for the existence of two ALS forms in the etiolated barley shoots. The two ALS forms (ALS I and ALS II) were separated well by heparin-affinity chromatography (Fig. 1). The two forms were similar in their 

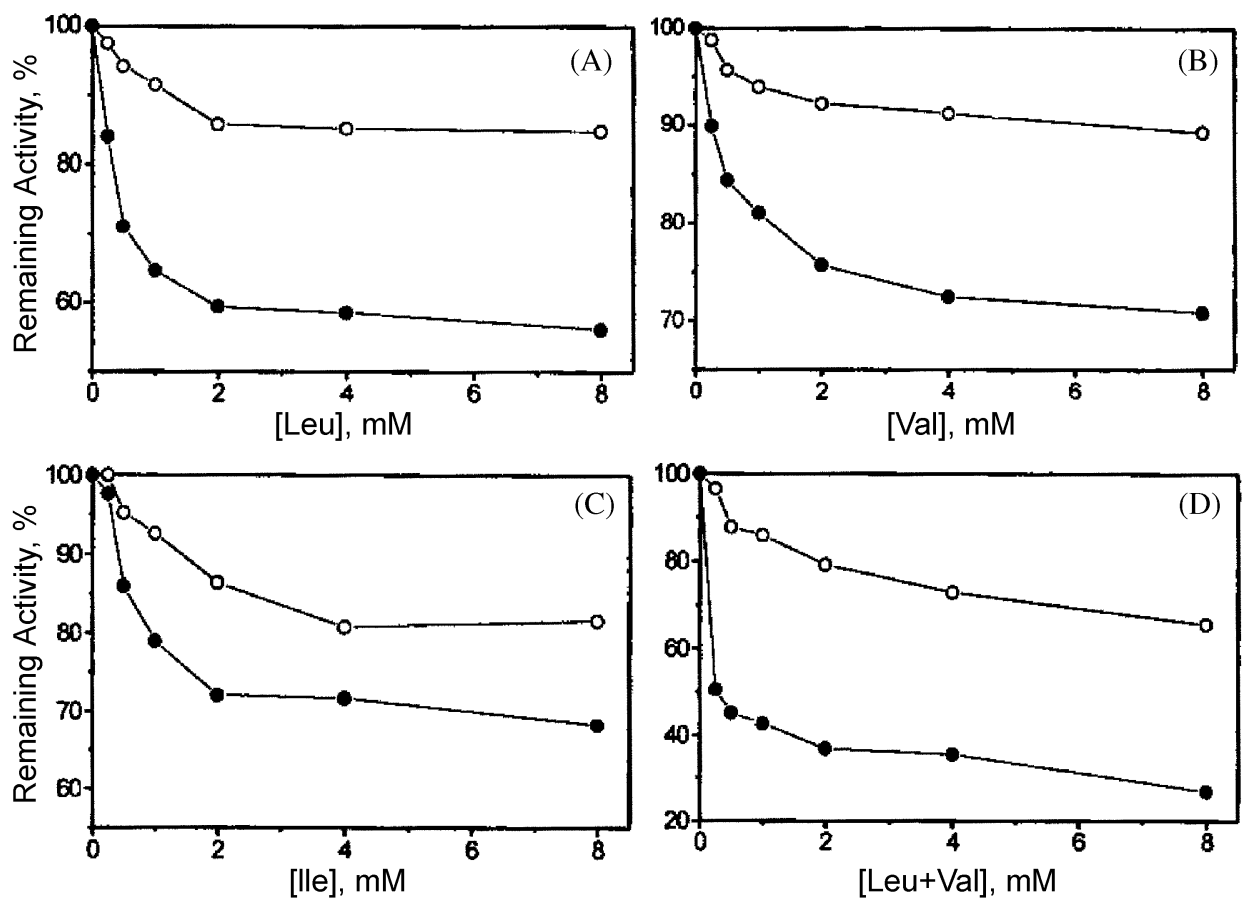

Fig. 7. Inhibition of ALS I and ALS II by leucine (A), valine (B), isoleucine (C), and leucine plus valine (1: 1) (D). ( $\bigcirc$, ALS I; ALS II).
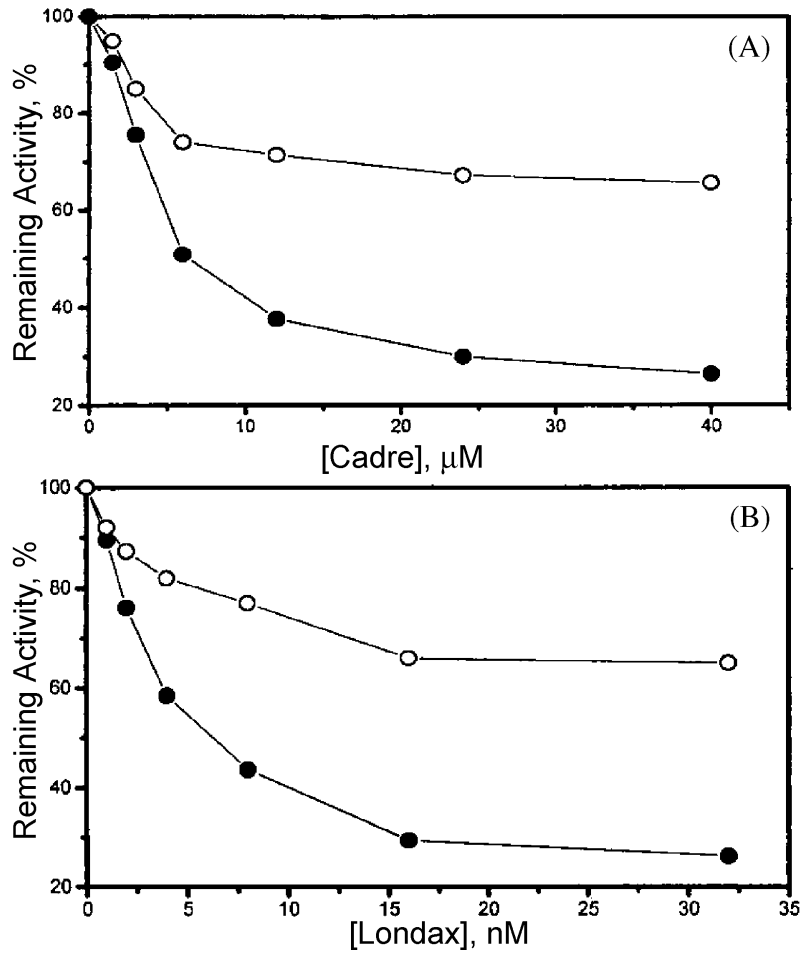

Fig. 8. Inhibition of ALS I $(\bigcirc)$ and $\operatorname{ALS}$ II ( $\mathbf{O})$ by Cadre (A) and Londax (B).

native molecular mass, but showed significant differences in $\mathrm{pI}$ and $\mathrm{pH}$ optima, as well as feedback regulation by the end products (Val, Leu, and Ile), and sensitivity to two classes of herbicides, (sulfonylurea and imidazilone). The molecular masses of native ALS I and ALS II were determined to be 248 $\mathrm{kDa}$ and $238 \mathrm{kDa}$ by nondenaturing gel electrophoresis and activity staining, respectively. The similar molecular mass of the two forms, 230-250 kDa, was confirmed by a Western blot analysis using an anti-ALS-antibody. Denaturing SDS-PAGE and a Western blot showed that the molecular masses of the ALS I and ALS II subunits were identical - $65 \mathrm{kDa}$. ALS II was more sensitive to feedback regulation by Leu, Val, Ile, and Leu plus Val than ALS I. Leu was the most effective amino acid inhibitor of both ALS forms. ALS II was more strongly inhibited by both sulfonylurea Londax and imidazolinone Cadre than ALS I. At this stage, our data are insufficient to define the origin of the two enzyme forms. Several possibilities for origin can be considered. One of the possibilities is that the two ALS types are different oligomeric forms of the same enzyme. However, this possibility could be excluded in the present case since molecular masses of native ALS I and ALS II appeared to be almost identical. Both ALS types are most likely tetramers of the $65 \mathrm{kDa}$ subunit. Another possibility is that, if there is a small regulatory subunit in plant ALS, then the two ALS forms may be due to the different compositions of the catalytic and regulatory subunits. In the present study, however, the small subunit was undetected by SDS-PAGE and following the Western blot. No direct evidence for the presence of a small regulatory subunit of plant ALS has been found. Thus, understanding the feedback inhibition of plant ALS is still unclear. Therefore, the results of this study suggest that the two ALS forms are likely two distinct isozymes. 
The isolation of the two ALS forms from Black Mexican Sweet corn cells was first reported by Singh et al. (1988). In this paper, the native molecular masses of the two ALS types were reported to be different, and it was suggested that they may be different oligomeric forms. Recently, Bekkaoui et al. (1991) reported the separation and characterization of two ALS forms from Brassica napus, which had an equal native molecular mass, but exhibited different properties with respect to the subunit structure, sensitivity to inhibition by chlorosulfuron, and feedback inhibition by branched-chain amino acids. Recently, Shin et al. (1999) also reported two ALS forms from peas, which showed significant differences in their native molecular mass and enzymatic properties.

Many microorganisms regulate the carbon flow in branched pathways by having multiple isozymes of a regulatory enzyme. Typically, each isozyme is feedback-regulated by the end product from a different pathway, diverging from a metabolic branch point. However, the two ALS forms in the present case do not fit this model, since both ALS I and ALS II are regulated in a similar pattern by the end products of the two pathways. The present data suggest that barley expresses two ALS forms, which exhibit different enzymatic properties. However, roles of the two ALS forms are still unclear, and a definitive interpretation for the two ALS forms will require further experiments to determine the peptide sequences of the two polypeptide subunits.

Acknowledgments This work was supported by a Korea Research Foundation Grant (KRF-2002-070-C00064).

\section{References}

Bekkaoui, F., Schorr, P. and Crosby, W. L. (1993) Acetolactate synthase from Barrasica napus: Immunological characterization and quaternary structure of the native enzyme. Physiol. Plant. 88, 475-484.

Bradford, M. M. (1976) A rapid and sensitive method for the quantitation of microgram quantities of protein utilizing the principle of protein-dye binding. Anal. Biochem. 72, 248-254.

Chipman, D., Barak, Z. and Schloss, J. V. (1998) Biosynthesis of 2-aceto-2-hydroxy acid: Acetolactate synthase and acetohydroxyacid synthases. Biochem. Biophys. Acta 1385, 401-419.

Chong, C.-K., Shin, H.-J., Chang, S.-I. and Choi, J.-D. (1999) The roles of tryptophanyl residues on tobacco acetolactate synthase Biochem. Biophys. Res. Commun. 259, 136-140.

Chong, C.-K. and Choi, J.-D. (2000) Amino acid residues conferring herbicide tolerance in tobacco acetolactate synthase. Biochem. Biophys. Res. Commun. 279, 462-467.

Davis, E. J., Blatt, J. M., Henderson, E. K., Whitalker, J. J. and Jackson, J. H. (1977) Valine-sensitive acetohydroxyacid synthase in Escherichia coli K-12: Unique regulation by multiple genetic sites. Mol. Gen. Genet. 156, 239-249.
Duggleby, R. G. and Pang, S. S. (2000) Acetohydroxyacid synthase. J. Biochem. Mol. Biol. 33, 1-36.

Eoyang, L. and Silverman, P. M. (1984) Purification and subunit composition of acetohydroxyacid synthase I from Escherichia coli K-12. J. Bacteriol. 157, 184-189.

Grimminger, H. and Umbarger, H. E. (1979) Acetohydroxyacid synthase I of Escherichia coli: Purification and properties. J. Bacteriol. 137, 846.

Joo, H. S. and Kim, S. S. (2001) Purification and characterization of the anabolic acetolactate synthase III from Serratia marceacens ATCC 75419. J. Biochem. Mol. Biol. 34, 244-249.

Kleschick, W. A., Costales, M. J., Dunbar, J. E., Meikla, R. W., Monte, W. T., Pearson, N. R., Snider, S. W. and Vinogradoff, Q. P. (1990) New herbicidal derivatives of 1,2,4-triazol [1.5-a] pyrimidine. Pestic. Sci. 29, 341-355.

Laemmli, U. K. (1979) Cleavage of structural proteins during the assembly of the head of bacteriophage T4. Nature 227, 620685.

LaRossa, R. A. and Schloss, J. V. (1984) The sulfonylurea herbicide sulfometuron methyl is extremely potent and selective inhibitor of acetolactate synthase in salmonella typhimurium. $J$. Biol. Chem. 259, 2753-8757.

Miflin, B. J. (1971) Cooperative feedback control of barley acetohydroxyacid synthase by leucine, isoleucine, and valine. Arch. Biochem. Biophys. 146, 542-550.

Namgoong, S. K., Lee, H. J., Kim, Y. S., Shin, J.-H., Che, J.-K., Jang, D. Y., Kim, G. S., Yoo, J. W., Kang, M.-K., Kil, M.-W., Choi, J.-D. and Chang, S.-I. (1999) Synthesis of the quinolinelinked triazolopyrimidine analogues and their interactions with the recombinant tobacco acetolactate synthase. Biochem. Biophy. Res. Commun. 258, 797-801.

Ray, T. B. (1984) Site of action of chlorsulfuron: inhibition of valine and isoleucine biosynthesis of plants. Plant Physiol. 75, 827-831.

Robertson, E. F., Dannelly, H. K., Malloy, P. J. and Reeves, H. C. (1987) Rapid isoelectric focusing in a vertical polyacrylamide minigel system. Anal. Biochem. 167, 290-294.

Shaner, D. L., Anderson, P. C. and Stidham, M. A. (1984) Imidazolinones: potent inhibitor of acetohydroxyacid synthase. Plant Physiol. 76, 545-546.

Schloss, J. V., Van Dyk, D. E., Vasta, J. F. and Kutny, R. M. (1985) Purification and properties of Salmonella typhymurium acetolactate synthase isozyme II from Escherichia coli HB101/ PDU9. Biochemistry 24, 4952-4959.

Shin, Y. S., Chong, C. K. and Choi, J. D. (1999) Separation and characterization of two forms of acetolactate synthase from etiolated pea seedlings. J. Biochem. Mol. Biol. 32, 393-398.

Singh, B. K., Stidham, M. A. and Shaner, D. L. (1988) Separation and characterization of two forms of acetohydroxyacid synthase from Black Mexican sweet corn cells. J. Chromatography 444, 251-261.

Yoon, T.-Y., Chung, S.-M., Chang, S.-I., Yoon M.-Y., Han, T.-R. and Choi, J.-D. (2002) Roles of lysine 219 and 255 residues in tobacco acetolactate synthase. Biochem. Biophys. Res. Commun. 293, 433-439.

Westerfeld, W. W. (1945) A colorimetric determination of blood acetoin. J. Biol. Chem. 161, 495-502. 